Gut, 1987, 28, S1, 103-107

\title{
Mucosal polyamine profile in normal and adapting (hypo and hyperplastic) intestine: effects of DFMO treatment
}

\author{
M HOSOMI, F LIRUSSI, N H STACE, S VAJA, G M MURPHY, \\ AND R H DOWLING
} From the Gastroenterology Unit, United Medical and Dental Schools of Guy's and St Thomas' Hospitals,
London

SUMMARY The polyamines, putrescine, spermidine, and spermine, are believed to play an important role in modulating normal and adaptive intestinal mucosal growth. Polyamine synthesis is rate limited by ornithine decarboxylase (ODC) and ODC activity is specifically inhibited by -difluoromethyl ornithine (DFMO). To assess the importance of polyamines in adaptive growth we first measured mucosal polyamine profiles at different sites in the normal rat intestine and compared the results with those obtained in adaptive hypoplasia (seven days parental nutrition, TPN), in the adaptive hyperplasia of two weeks after $90 \%$ small bowel resection (SBR) or pancreatico biliary diversion (PBD). We then examined the effects of DFMO (2\% in drinking water, daily from two days before surgery) on the polyamine concentrations and the adaptive response to PBD. The hyperplasia of SBR and PBD was associated with increases in all the polyamine concentrations particularly putrescine. TPN induced a modest degree of hypoplasia and little change in polyamine synthesis resulting in subnormal polyamine concentrations and significantly inhibited the mucosal adaptive response. Changes in polyamine metabolism are important in intestinal mucosal adaptation and by controlling these changes adaptive growth can be controlled.

The ability of the small intestine to adapt in structure and function is now a well established phenomenon. Since the first reports of intestinal adaptation at the turn of the century ${ }^{12}$ much information has been accumulated about the various factors which can influence it. Only in recent years, however, have events within the mucosal cells themselves, received any attention. In particular, the small polyvalent cations, the polyamines (Fig 1) have been implicated as playing a major role in the intracellular events associated with the adaptive response. ${ }^{3-5}$

In this report we consider why changes in polyamine concentrations might be thought essential in the process of adaptation. And we look at those changes induced in the normal intestinal polyamine profile during adaptive responses both hyperplastic and hypoplastic. To do this, we measured polyamine levels in rat intestinal mucosa after seven days total parenteral nutrition (the hypoplastic model) ${ }^{6}$ and at

Address for correspondence: Professor R Hermon Dowling, 18th Floor, Guy's Tower, Guy's Hospital, London Bridge, London SE1 9RT.
14 days after pancreaticobiliary diversion or $90 \%$ small bowel resection (two hyperplastic models). ${ }^{6}$ Finally we consider what happens in these models if we interfere with polyamine metabolism directly, by administering an inhibitor of polyamine biosynthesis.

But first, what is there about polyamines which makes them likely candidates in the cellular control of the adaptive response? From the point of view of chemical structure, the polyamines may be regarded as polycations in which the positive charges are separated by regular spacing (Fig 1). It is presumably because of this cationic distribution that they have a high affinity for nucleic acids. ${ }^{7}$ Although the precise function of polyamines in mammalian cells has yet to be defined, they appear to be essential for cell growth and differentiation ${ }^{8}$ and to be capable of interacting with many metabolic processes. For example, they can interfere with steroid metabolism ${ }^{2}$ and lipid peroxidation. ${ }^{10}$ Little is known, however, of the relative importance of the different polyamines in these functions. This subject has recently been reviewed by Pegg. ${ }^{11}$ 


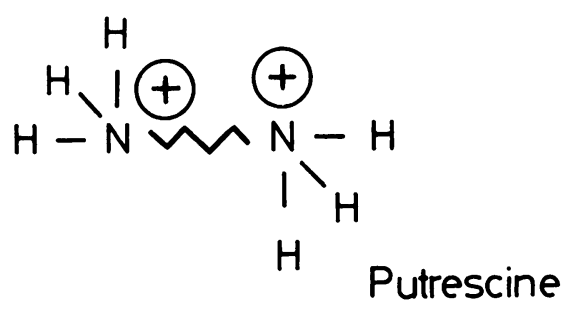<smiles></smiles>

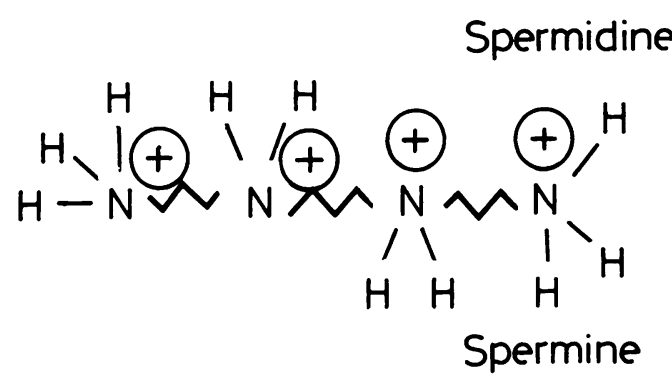

Fig. 1 Growth associated polyamines in man.

If polyamines do play an essential role in the adaptive response, what characteristics would we expect them to show? In a hyperplastic response we would expect the polyamine concentrations to be increased not only if expressed in amounts per unit length of tissue but more importantly when expressed per unit DNA.

In a hypoplastic response we would expect no change or even a decrease in polyamine concentrations. Furthermore, if we could inhibit the synthesis of polyamines by an independent means (independent of the adaptation-provoking mechanism) then we would expect the hyperplastic adaptive response to be severely curtailed. One way of reducing polyamine synthesis is to administer an inhibitor of the rate limiting enzyme, ornithine decarboxylase. Such an inhibitor is difluoro methyl ornithine (DFMO) which has frequently been used as a specific inhibitor of polyamine biosynthesis (Fig 2). ${ }^{12}$

\section{Methods}

MODELS

Our methods of analysis and models of hypo- and hyperplasia have been fully described elsewhere. ${ }^{613}$ In

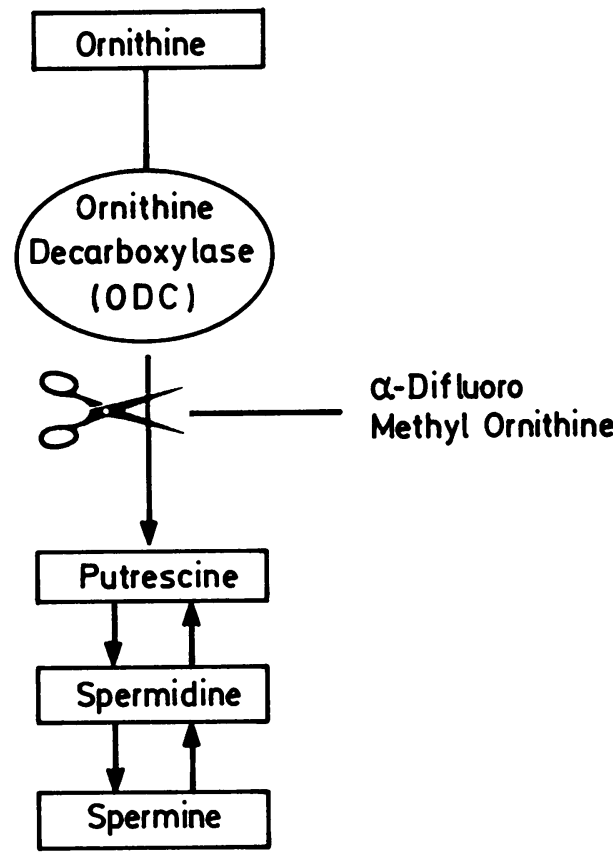

Fig. 2 The inhibitory action of -difluoro methyl ornithine on ornithine decarboxylase.

brief, we measured polyamine concentrations at different sites in the normal rat intestine (duodenum, $5 \times 10 \mathrm{~cm}$ segments of jejunum, $5 \times 10 \mathrm{~cm}$ segments of ileum, and colon) and compared the results with those obtained in the total parenteral nutrition model (TPN), in the pancreatico-biliary diversion model (PBD) and in the small bowel resection model (SBR). We also examined the effect of DFMO (administered as a $2 \%$ solution in drinking water, daily from two days before surgery) on both the polyamine concentrations and the common indices of adaptation of PBD.

\section{Results and discussion}

The first question then is - did the models we used show the expected adaptive response?

In the group of animals receiving total parenteral nutrition, the indices of mucosal mass (wet weight, protein, and DNA per $10 \mathrm{~cm}$ mucosal length) were all some $20 \%$ less than those in the unoperated, normally fed, control group. Although by no means dramatic, these results were consistent with the notion that just seven days TPN induced a modest degree of hypoplasia.

In the hyperplastic groups, although the results were somewhat variable they were much more impressive than those in the TPN group. Compared 

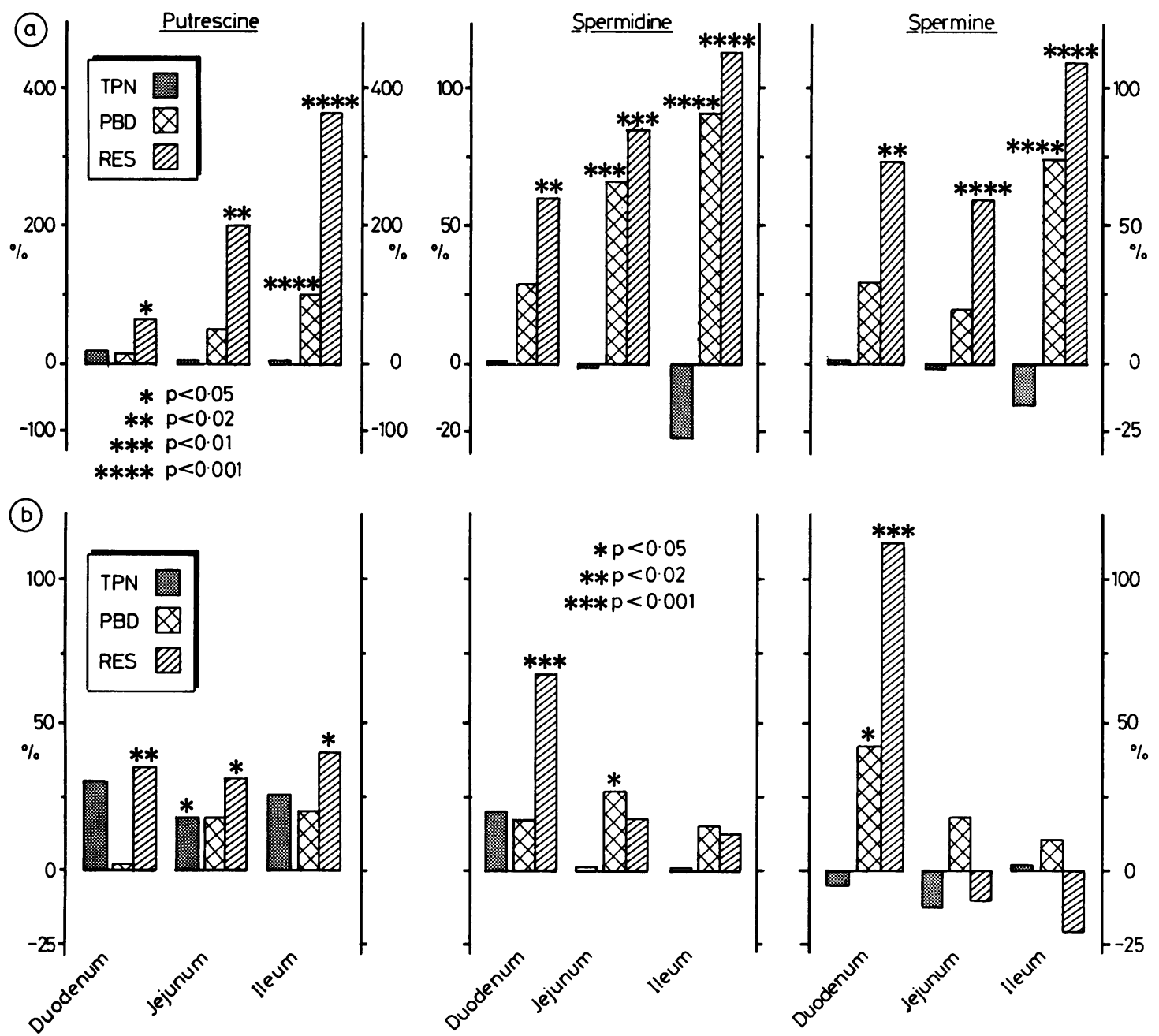

Fig. 3(a) \% Changes in polyamine concentrations per unit length mucosa (mean) on the left TPN, the hypoplastic model, the two hyperplastic models $P B D$ and resection on the right ${ }^{*} p<0.05,{ }^{* *} p<0.02,{ }^{* * *} p<0 \cdot 01,{ }^{* * * *} p<0.001$.

Fig. 3(b) \% Changes in polyamine concentrations per unit weight mucosal DNA (mean $\pm S E M)$ : designation as 3(a) ${ }^{*} p<0.05,{ }^{* *} p<0.02,{ }^{* * *} p<0.001$.

with those of the animals in the transected control group, the indices of small intestinal mucosa mass were increased in the PBD group by $20-60 \%$ and in the resected group by $60-160 \%$.

What then of the polyamines? Hyperplasia was associated with increases in the concentrations of putrescine and spermidine at the different intestinal sites (Fig 3). As might have been expected, the largest increases were found associated with the hyperplasia induced by resection. The relative increases in putrescine concentrations were greater than those in spermidine or spermine. Indeed the amount of spermine per unit DNA was reduced in both jejunum and ileum when compared with that in the transected controls (Fig 3b). In contrast, polyamine concentrations throughout the intestine appeared to be little changed by TPN, although when expressed per mg DNA putrescine concentrations were increased at all three sites (Fig 3b).

So pancreaticobiliary diversion induces a hyperplastic adaptive response which is associated with an increase in polyamines. What happens to that adaptive response if we feed the animals the inhibitor of polyamine synthesis, DFMO? The results are illustrated in Figure 4. In both the jejunum and the ileum, the adaptive response to PBD is inhibited by 


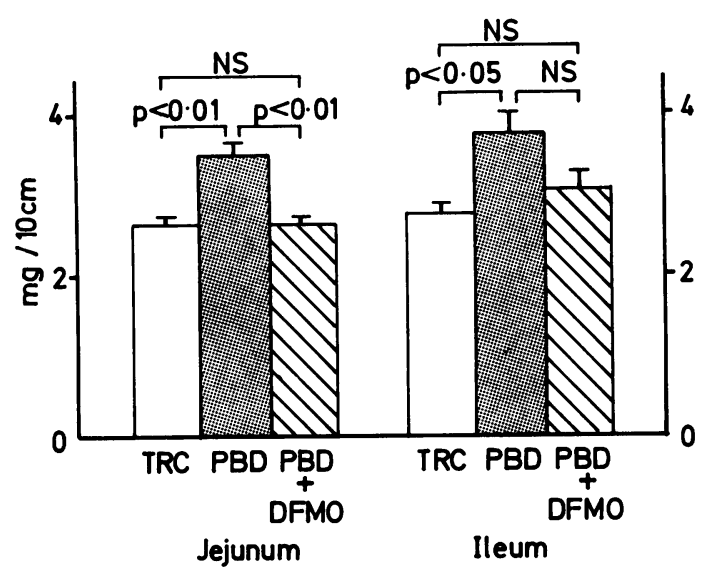

Fig. 4 Effects of DFMO on the adaptive response (mg DNA/10 cm length) in the mucosa of jejunum and ileum to $P B D$.
DFMO. And as shown in Figure 5, with the exception of spermine, polyamine concentrations were lower in the tissues of those animals fed DFMO than in either the transected controls or the 'hyperplastic' PBD group. The results, however, were not always consistent: the hyperplasia induced by PBD in this particular group of animals was not invariably associated with an increase in polyamine concentrations. Nevertheless, it is clear that administration of DFMO results in a reduction in mucosal polyamine concentrations and in turn that this reduction is associated with a diminished adaptive response in established models of hyperplasia.

In conclusion, these results support the concept that changes in polyamine metabolism are important in intestinal mucosal adaptation. They also suggest that by manipulating polyamine concentrations, adaptive mucosal growth may be manipulated and controlled.
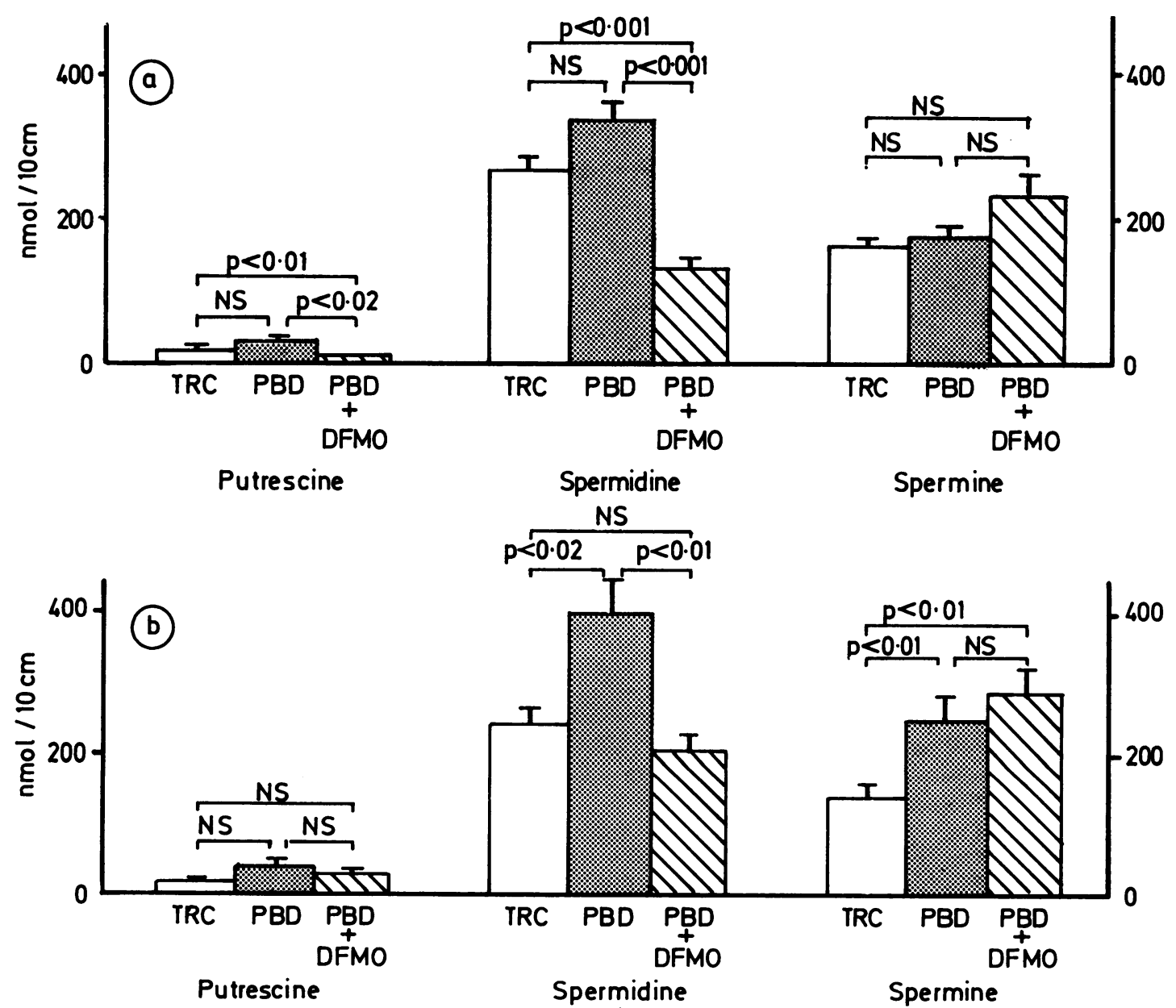

Fig. 5(a) Effect of DFMO on the concentrations (nmol/10 cm) of polyamines in the jejunal mucosa.

Fig. 5(b) Effect of DFMO on the concentrations (nmol/10 cm) of polyamine in the ileal mucosa. 
This work was supported by the Hyogo College of Medicine (MH) and the Special Trustees, Guy's Hospital. Thanks are also due to Marcia Birkin who typed the manuscript.

\section{References}

1 Senn N. An experimental contribution to intestinal surgery with special reference to the treatment of intestinal obstruction II. Enterectomy. Ann Surg 1888; 7: 99-105.

2 Flint JM. The effect of extensive resections of the small intestine. Bull John Hopkins Hosp 1912; 23: 127-44.

3 Luk GD, Marton LJ, Baylin SB. Ornithine decarboxylase is important in intestinal mucosal maturation and recovery from injury in rats. Science 1980; 210: 195-8.

4 Luk GD, Baylin SB. Polyamines and intestinal growth increased polyamine biosynthesis after jejunectomy. $\mathrm{Am}$ J Physiol 1983; 245: G656-60.

5 Porter CW, Dworaczyk D, Ganis B, Weiser MM. Polyamines and biosynthetic enzymes in the rat intestinal mucosa and the influence of methylglyoxal-bio guaryl hydrazone. Cancer Res 1980; 40: 2330-5.

6 Hosomi M, Stace NH, Lirussi F, Smith SM, Murphy
GM, Dowling RH. The role of polyamines in intestinal adaptation in the rat. Eur J Clin Invest 1987 (In press.)

7 Raina A, Janne J. Physiology of the natural polyamines, putrescine, spermidine and spermine. Med Biol 1975; 53: $121-47$

8 Pegg AE, McCann PP. Polyamine metabolism and function. Am J Physiol 1982; 243: C212-21.

9 Jellinick PH, Perry G. Effect of polyamines on the metabolism of $16-14 \mathrm{C}$ estradiol by rat liver microsomes. Biochim Biophys Acta 1967; 137: 367-74.

10 Kitada W, Igavashi K, Hirose S, Kitagawa H. Inhibition of polyamines of lipid peroxide formation in rat liver microsomes. Biochim Biophys Res Comm 1979; 87: 388-94.

11 Pegg AE Recent advances in the biochemistry of polyamines in eukaryotes. Biochem $J$ 1986; 234 : 24962.

12 Luk GD, Boylin SB. Inhibition of intestinal epithelial DNA Synthesis and adaptive hyperplasia after jejunectomy in the rat by suppression of polyamine biosynthesis. J Clin Invest 1984; 74: 698-704.

13 Hosomi M, Smith SM, Murphy GM, Dowling RH. Polyamine distribution in the rat intestinal mucosa. J Chromatogr 1986; 375: 267-275. 